\title{
Imaging in urolithiasis
}

\author{
Peggy Yen MD, Mark O. Baerlocher MD
}

\section{Non-contrast-enhanced computed tomography is the "gold standard" for the evaluation of renal colic}

Computed tomography $(\mathrm{CT})$ can be performed rapidly and can detect radiolucent stones, except for drug-induced stones. It is preferred over intravenous pyelography or urography because it is more sensitive and does not require the use of intravenous contrast medium. Stone size and burden, and the degree of urinary obstruction can be directly measured. Up to $90 \%$ of stones less than $5 \mathrm{~mm}$ in diameter, particularly if located in the distal ureter, will pass spontaneously. ${ }^{1}$ Stones greater than $5 \mathrm{~mm}$ usually require urologic intervention. The density of stones can also be determined using Hounsfield units (HU); stones with a density greater than $1000 \mathrm{HU}$ respond less well to lithotripsy. ${ }^{1}$

\section{Ultrasonography is the initial in- vestigation of choice in pregnant women and in children}

The sensitivity of ultrasonography depends on the size and location of the stone and the body habitus or presence of overlying bowel gas. ${ }^{3}$ Adequate hydration aids in the detection of the calculus. When ultrasonography yields inconclusive results, limited sequence intravenous urography or low-dose non-contrast-enhanced CT can be considered for pregnant patients; exposures required to image the enlarged maternal abdomen with conventional radiography can increase the radiation dose level to that of $\mathrm{CT} .{ }^{4,5}$ In children, plain radiography is the second choice to ultrasonography, followed by low-dose noncontrast-enhanced CT $\mathrm{CT}^{5}$ (see Appendix 1, at www.cmaj.ca/lookup/suppl/doi :10.1503/cmaj.101638/-/DC1).

\section{Radiographic follow-up is helpful for specific groups of patients}

Because complications of urolithiasis include infection and obstruction that may lead to septicemia or irreversible parenchymal damage, radiographic follow-up is useful in patients with multiple episodes of symptomatic stone disease, those who elect conservative management and those who have undergone a urologic intervention. ${ }^{1,3} \mathrm{~A}$ patient who is passing a stone spontaneously should be followed up every one or two weeks for three to four weeks to follow progression and monitor for obstruction. ${ }^{\text {If }}$ there is a known radiopaque stone, plain radiography can be performed two to four weeks after any surgical intervention to ensure no clinically significant fragments remain. ${ }^{3}$
Plain radiography can be useful in investigating renal colic in patients with a history of radiopaque stones

Plain radiography of the kidneys, ureters and bladder is a reasonable initial investigation in patients with a history of radiopaque stones who present with renal colic. The mean size of the stone detected with plain radiography is $4.2 \mathrm{~mm}$; the mean size missed is $3.1 \mathrm{~mm} .^{2}$ For the $10 \%$ of patients with known radiolucent calculi, CT or ultrasonography are better diagnostic alternatives. ${ }^{3}$

Radiographic screening can monitor stone burden

Annual plain radiography is useful in patients who have asymptomatic, radiopaque stones and those who form recurrent stones to ensure stone burden is not increasing. ${ }^{3}$

\section{References}

1. Parmar MS. Clinical review: kidney stones. BMJ 2004;328:1420-4.

2. Jindal G, Ramchandani P. Acute flank pain secondary to urolithiasis: radiologic evaluation and alternate diagnoses. Radiol Clin North Am 2007;45:395-410.

3. Dhar M, Denstedt JD. Imaging in diagnosis, treatment, and follow-up of stone patients. Adv Chronic Kidney Dis 2009;16:39-47.

4. Srirangam SJ, Hickerton B, Cleynenbreugel BV. Management of urinary calculi in pregnancy: a review. J Endourol 2008;22:867-75.

5. Hiorns MP. Imaging of urinary tract lithiasis: Who, when and how? Pediatr Radiol 2008;38(Suppl 3): S497-500.

Competing interests: None declared.

This article has been peer reviewed.

Affiliations: From the Radiology Residency Training Program (Yen), Dalhousie University, Halifax, NS; and the Department of Interventional Radiology (Baerlocher), University of California San Diego, San Diego, Calif.

Correspondence to: Dr. Peggy Yen, peggy.yen @ dal.ca

CMAJ 2011. DOI:10.1503/cmaj.101638
CMAJ invites submissions to "Five things to know about ..." Submit manuscripts online at http://mc.manuscriptcentral.com/cmaj 